\title{
Approximation numbers of composition operators on the Hardy space of the infinite polydisk
}

\author{
Daniel Li, Hervé Queffélec, L. Rodríguez-Piazza \\ September 17, 2018
}

\begin{abstract}
We study the composition operators of the Hardy space on $\mathbb{D}^{\infty} \cap \ell_{1}$, the $\ell_{1}$ part of the infinite polydisk, and the behavior of their approximation numbers.
\end{abstract}

\section{Introduction}

Recently, in [2], we investigated approximation numbers $a_{n}\left(C_{\varphi}\right), n \geq 1$, of composition operators $C_{\varphi}, C_{\varphi}(f)=f \circ \varphi$, on the Hardy or Bergman spaces $H^{2}(\Omega), B^{2}(\Omega)$ over a bounded symmetric domain $\Omega \subseteq \mathbb{C}^{d}$. Assuming that $\varphi(\Omega)$ has non-empty interior, one of the main results of this study was the following theorem.

Theorem $1.1([2])$. Let $C_{\varphi}: H^{2}(\Omega) \rightarrow H^{2}(\Omega)$ be compact. Then:

1) we always have $a_{n}\left(C_{\varphi}\right) \geq c \mathrm{e}^{-C n^{1 / d}}$ where $c, C$ are positive constants;

2) if $\Omega$ is a product of balls and if $\varphi(\Omega) \subseteq r \Omega$ for some $r<1$, then:

$$
a_{n}\left(C_{\varphi}\right) \leq C \mathrm{e}^{-c n^{1 / d}} .
$$

As a result, the minimal decay of approximation numbers is slower and slower as the dimension $d$ increases, which might lead one to think that, in infinite-dimension, no compact composition operators can exist, since their approximation numbers will not tend to 0 . After all, this is the case for the Hardy space of a half-plane, which supports no compact composition operator ([12], Theorem 3.1; in [9], it is moreover proved that $\left\|C_{\varphi}\right\|_{e}=\left\|C_{\varphi}\right\|$ as soon as $C_{\varphi}$ is bounded; see also [15] for a necessary and sufficient condition for $H^{2}(\Omega)$ has compact composition operators, where $\Omega$ is a domain of $\mathbb{C}$ ). We will see that this is not quite the case here, even though the decay will be severely limited. In particular, we will never have a decay of the form $C \mathrm{e}^{-c n^{\delta}}$ for some $c, C, \delta>0$. 


\section{$2 \quad$ Framework and reminders}

\subsection{Hardy spaces on $\mathbb{D}^{\infty}$}

Let $\mathbb{T}=\partial \mathbb{D}$ be the unit circle of the set of complex numbers. We consider $\mathbb{T}^{\infty}$ and equip it with its Haar measure $m$. This is a compact Abelian group with dual $\mathbb{Z}^{(\infty)}$, the set of eventually zero sequences $\alpha=\left(\alpha_{j}\right)_{j \geq 1}$ of integers. We denote $L_{\mathbb{N}(\infty)}^{2}\left(\mathbb{T}^{\infty}\right)$ the Hilbert subspace of $L^{2}\left(\mathbb{T}^{\infty}\right)$ formed by the functions $f$ whose Fourier spectrum is contained in $\mathbb{N}^{(\infty)}$ :

$$
\widehat{f}(\alpha):=\int_{\mathbb{T}^{\infty}} f(z) \bar{z}^{\alpha} d m(z)=0 \quad \text { if } \alpha \notin \mathbb{N}^{(\infty)} .
$$

The set $E:=\mathbb{N}^{(\infty)}$ is called the narrow cone of Helson, and we also denote $L_{\mathbb{N}(\infty)}^{2}\left(\mathbb{T}^{\infty}\right)=L_{E}^{2}\left(\mathbb{T}^{\infty}\right)$. Any element of that subspace can be formally written as:

$$
f=\sum_{\alpha \geq 0} c_{\alpha} e_{\alpha} \quad \text { with } c_{\alpha}=\widehat{f}(\alpha) \text { and } \quad \sum_{\alpha \geq 0}\left|c_{\alpha}\right|^{2}<\infty .
$$

Here, $\left(e_{\alpha}\right)_{\alpha \in \mathbb{Z}^{(\infty)}}$ is the canonical basis of $L^{2}\left(\mathbb{T}^{\infty}\right)$ formed by characters, and accordingly $\left(e_{\alpha}\right)_{\alpha \in \mathbb{N}(\infty)}$ is the canonical basis of $L_{E}^{2}\left(\mathbb{T}^{\infty}\right)$.

Now we consider $\Omega_{2}=\mathbb{D}^{\infty} \cap \ell_{2}$.

Any $f \sim \sum_{\alpha>0} c_{\alpha} e_{\alpha} \in L_{E}^{2}\left(\mathbb{T}^{\infty}\right)$ defines an analytic function on the infinitedimensional Reinhardt domain $\Omega_{2}$ by the formula:

$$
f(z)=\sum_{\alpha \geq 0} c_{\alpha} z^{\alpha}
$$

where the series is absolutely convergent for each $z=\left(z_{j}\right)_{j \geq 1} \in \Omega_{2}$, as the pointwise product of two square-summable sequences. Indeed, using an Euler type formula, we get for $z \in \Omega_{2}$ :

$$
\sum_{\alpha \geq 0}\left|z^{\alpha}\right|^{2}=\prod_{j=1}^{\infty}\left(1-\left|z_{j}\right|^{2}\right)^{-1}<\infty,
$$

and hence, by the Cauchy-Schwarz inequality:

$$
\sum_{\alpha \geq 0}\left|c_{\alpha} z^{\alpha}\right| \leq\left(\sum_{\alpha \geq 0}\left|c_{\alpha}\right|^{2}\right)^{1 / 2}\left(\sum_{\alpha \geq 0}\left|z^{\alpha}\right|^{2}\right)^{1 / 2}<\infty .
$$

If $\alpha \in E$ and $z \in \Omega_{2}$, we have set, as usual, $z^{\alpha}=\prod_{j \geq 1} z_{j}^{\alpha_{j}}$.

This shows that $L_{E}^{2}\left(\mathbb{T}^{\infty}\right)$ can be identified with $H^{2}\left(\Omega_{2}\right)$, the Hardy-Hilbert space of analytic functions $f(z)=\sum_{\alpha \geq 0} c_{\alpha} z^{\alpha}$ on $\Omega_{2}$ with

$$
\|f\|^{2}:=\sum_{\alpha \geq 0}\left|c_{\alpha}\right|^{2}<\infty
$$


This setting is customary in connection with Dirichlet series (see [7]).

In this paper, for a technical reason appearing below in the proof of Proposition 2.5. we will consider, instead of $\Omega_{2}=\mathbb{D}^{\infty} \cap \ell_{2}$, the sub-domain:

$$
\Omega=\mathbb{D}^{\infty} \cap \ell_{1},
$$

i.e. the open subset of $\ell^{1}$ formed by the sequences:

$$
z=\left(z_{n}\right)_{n \geq 1} \quad \text { such that }\left|z_{n}\right|<1, \forall n \geq 1, \quad \text { and } \quad \sum_{n=1}^{\infty}\left|z_{n}\right|<\infty,
$$

and the restrictions to $\Omega$ of the functions $f \in H^{2}\left(\Omega_{2}\right)$. We denote $H^{2}(\Omega)$ the space of such restrictions.

Hence $f \in H^{2}(\Omega)$ if and only if:

$$
f(z)=\sum_{\alpha \geq 0} c_{\alpha} z^{\alpha} \quad \text { with } z \in \Omega,
$$

and $\|f\|^{2}:=\sum_{\alpha \geq 0}\left|c_{\alpha}\right|^{2}<\infty$.

We now identify the space $L_{E}^{2}\left(\mathbb{T}^{\infty}\right)$ with the space $H^{2}(\Omega)$.

We more generally define Hardy spaces $H^{p}(\Omega)$, for $1 \leq p<\infty$, in the usual way:

$$
H^{p}=H^{p}(\Omega)=\left\{f: \Omega \rightarrow \mathbb{C} ;\|f\|_{p}<\infty\right\},
$$

where $f$ is analytic in $\Omega$ and $\|f\|_{p}=\sup _{0<r<1} M_{p}(r, f)=\lim _{r \rightarrow 1^{-}} M_{p}(r, f)$ with:

$$
M_{p}(r, f)=\left(\int_{\mathbb{T}^{\infty}}|f(r z)|^{p} d m(z)\right)^{1 / p}, \quad 0<r<1 .
$$

We have $\|f\|=\|f\|_{2}$. Moreover, $H^{q}$ contractively embeds into $H^{p}$ for $p<q$.

\subsection{Singular numbers}

We begin with a reminder of operator-theoretic facts. We recall that the approximation numbers $a_{n}(T)=a_{n}$ of an operator $T: H \rightarrow H$ (with $H$ a Hilbert space) are defined by:

$$
a_{n}=\inf _{\operatorname{rank} R<n}\|T-R\| .
$$

According to a 1957's result of Allahverdiev (see [3], page 155), we have $a_{n}=s_{n}$, the $n$-th singular number of $T$. We also recall a basic result due to $\mathrm{H}$. Weyl and one obvious consequence:

Theorem 2.1. Let $T: H \rightarrow H$ be a compact operator with eigenvalues $\left(\lambda_{n}\right)$ rearranged in decreasing order and singular numbers $\left(a_{n}\right)$. Then:

$$
\prod_{j=1}^{n}\left|\lambda_{j}\right| \leq \prod_{j=1}^{n} a_{j} \quad \text { for all } n \geq 1 .
$$

As a consequence:

$$
\left|\lambda_{2 n}\right|^{2} \leq a_{1} a_{n} .
$$




\subsection{Spectra of projective tensor products}

The following operator-theoretic result will play a basic role in the sequel. Let $E_{1}, \ldots, E_{n}$ be Banach spaces and let $E=\otimes_{i=1}^{n} E_{i}$ their projective tensor product (the only tensor product we shall use). If $T_{i} \in \mathcal{L}\left(E_{i}\right)$, we define as usual their projective tensor product $T=\otimes_{i=1}^{n} T_{i} \in \mathcal{L}(E)$ by its action on the atoms of $E$, namely:

$$
T\left(\otimes_{i=1}^{n} x_{i}\right)=\otimes_{i=1}^{n} T_{i}\left(x_{i}\right) .
$$

Denote in general $\sigma(x)$ the spectrum of $x \in \mathcal{A}$ where $\mathcal{A}$ is a unital Banach algebra. We recall ([13], chap.11, Theorem 11.23) the following result.

Lemma 2.2. Let $\mathcal{A}$ be a unital Banach algebra, and $x_{1}, \ldots, x_{n}$ be pairwise commuting elements of $\mathcal{A}$. Then:

$$
\sigma\left(x_{1} \cdots x_{n}\right) \subseteq \prod_{i=1}^{n} \sigma\left(x_{i}\right)
$$

Here, $\prod_{i=1}^{n} \sigma\left(x_{i}\right)$ is the product in the Minkowski sense, namely:

$$
\prod_{i=1}^{n} \sigma\left(x_{i}\right)=\left\{\prod_{i=1}^{n} \lambda_{i}: \lambda_{i} \in \sigma\left(x_{i}\right)\right\} .
$$

As a consequence, we then have the following lemma due to Schechter, which we prove under a weakened form, sufficient here, and which is indeed already in [1] (we just add a few details because this is a central point in our estimates).

Lemma 2.3. Let $F$ be a Banach space, $T_{1}, \ldots, T_{n} \in \mathcal{L}(F)$ and $T=\otimes_{i=1}^{n} T_{i}$. Then $\sigma(T) \subset \prod_{i=1}^{n} \sigma\left(T_{i}\right)$.

Proof. To save notation, we assume $n=2$. Let $x_{1}=T_{1} \otimes I_{2}$ and $x_{2}=I_{1} \otimes T_{2}$ where $I_{i}$ is the identity of $E_{i}$. Clearly,

$$
x_{1} x_{2}=x_{2} x_{1}=T_{1} \otimes T_{2}=T \quad \text { and } \quad \sigma\left(x_{i}\right)=\sigma\left(T_{i}\right)
$$

where the spectrum of $x_{i}$ is in the Banach algebra $\mathcal{L}(E)$ and that of $T_{i}$ in $\mathcal{L}\left(E_{i}\right)$. Lemma 2.2 now gives:

$$
\sigma(T)=\sigma\left(x_{1} x_{2}\right) \subseteq \sigma\left(x_{1}\right) \sigma\left(x_{2}\right)=\sigma\left(T_{1}\right) \sigma\left(T_{2}\right),
$$

hence the result.

\subsection{Schur maps and composition operators}

We now pass to some general facts on composition operators $C_{\varphi}$, defined by $C_{\varphi}(f)=f \circ \varphi$, associated with a Schur map, namely a non-constant analytic self-map $\varphi$ of $\Omega$. We say that $\varphi$ is a symbol for $H^{2}(\Omega)$ if $C_{\varphi}$ is a bounded linear operator from $H^{2}(\Omega)$ into itself.

The differential $\varphi^{\prime}(a)$ of $\varphi$ at some point $a \in \Omega$ is a bounded linear map $\varphi^{\prime}(a): \ell^{1} \rightarrow \ell^{1}$. 
Definition 2.4. The symbol $\varphi$ is said to be truly infinite-dimensional if the differential $\varphi^{\prime}(a)$ is an injective linear map from $\ell^{1}$ into itself for at least one point $a \in \Omega$.

In finite dimension, this amounts to saying that $\varphi(\Omega)$ has non-void interior.

We have the following general result.

Proposition 2.5. Let $\left(\varphi_{j}\right)_{j \geq 1}$ be a sequence of analytic self-maps of $\mathbb{D}$ such that $\sum_{j \geq 1}\left|\varphi_{j}(0)\right|<\infty$. Then, the mapping $\varphi: \Omega \rightarrow \mathbb{C}^{\infty}$ defined by the formula $\varphi(z)=\left(\varphi_{j}\left(z_{j}\right)\right)_{j \geq 1}$ maps $\Omega$ to itself and is a symbol for $H^{2}(\Omega)$.

Proof. First, the Schwarz inequality:

$$
\left|\varphi_{j}\left(z_{j}\right)-\varphi_{j}(0)\right| \leq 2\left|z_{j}\right|
$$

shows that $\varphi(z) \in \Omega$ when $z \in \Omega$. To see that $\varphi$ is moreover a symbol for $H^{2}(\Omega)$, we use the fact $([8)$ that:

$$
\left\|C_{\varphi_{j}}\right\| \leq \sqrt{\frac{1+\left|\varphi_{j}(0)\right|}{1-\left|\varphi_{j}(0)\right|}} .
$$

Now, by the separation of variables and Fubini's theorem, we easily get:

$$
\left\|C_{\varphi}\right\| \leq \prod_{j=1}^{\infty}\left\|C_{\varphi_{j}}\right\|<\infty .
$$

As $\sum_{j \geq 1}\left|\varphi_{j}(0)\right|<\infty$, by hypothesis, the infinite product

$$
\prod_{j \geq 1} \sqrt{\frac{1+\left|\varphi_{j}(0)\right|}{1-\left|\varphi_{j}(0)\right|}}
$$

converges and, in view of (2.2) and (2.3),$C_{\varphi}$ is bounded.

We also have the following useful fact.

Lemma 2.6. The automorphisms of $\Omega$ act transitively on $\Omega$ and define bounded composition operators on $H^{2}(\Omega)$.

Proof. Let $a=\left(a_{j}\right)_{j} \in \Omega$ and let $\Psi_{a}: \Omega \rightarrow \mathbb{C}^{\infty}$ be defined by:

$$
\Psi_{a}(z)=\left(\Phi_{a_{j}}\left(z_{j}\right)\right)_{j \geq 1}
$$

where in general $\Phi_{u}: \mathbb{D} \rightarrow \mathbb{D}$ is defined by $\Phi_{u}(z)=(z-u) /(1-\bar{u} z)$. The Schwarz lemma gives $\left|\Phi_{a_{j}}\left(z_{j}\right)+a_{j}\right| \leq 2\left|z_{j}\right|$, and shows that $\Psi_{a}$ maps $\Omega$ to itself. Clearly, $\Psi_{a}$ is an automorphism of $\Omega$ with inverse $\Psi_{-a}$ and $\Psi_{a}(a)=0$. The fact that the composition operator $C_{\Psi_{a}}$ is bounded on $H^{2}(\Omega)$ is a consequence of Proposition 2.5. 


\section{Spectrum of compact composition operators}

We begin with the following definition, following [10].

Definition 3.1. Let $\underline{\varphi: \Omega} \rightarrow \Omega$ be a truly infinite-dimensional symbol. We say that $\varphi$ is compact if $\overline{\varphi(\Omega)}$ is a compact subset of $\Omega$.

We then have the following result.

Lemma 3.2. If $\varphi: \Omega \rightarrow \Omega$ is a compact mapping, then:

1) $C_{\varphi}: H^{2}(\Omega) \rightarrow H^{2}(\Omega)$ is bounded and moreover compact.

2) If $a \in \Omega$ a fixed point of $\varphi, \varphi^{\prime}(a) \in \mathcal{L}\left(\ell^{1}\right)$ is a compact operator.

Proof. 1) follows from a H. Schwarz type criterion via an Ascoli-Montel type theorem: every sequence $\left(f_{n}\right)$ of $H^{2}(\Omega)$ bounded in norm contains a subsequence which converges uniformly on compact subsets of $\Omega$. Indeed, we have the following (4, chap. 17, p. 274): if $A$ is a locally bounded set of holomorphic functions on $\Omega$, then $A$ is locally equi-Lipschitz, namely every point $a \in \Omega$ has a neighourhood $U \subset \Omega$ such that:

$$
z, w \in U \quad \text { and } \quad f \in A \quad \Longrightarrow \quad|f(z)-f(w)| \leq C_{A, U}\|z-w\| .
$$

The Ascoli-Montel theorem easily follows from this. Then, if $f_{n} \in H^{2}(\Omega)$ converges weakly to 0 , it converges uniformly to 0 on compact subsets of $\Omega$; in particular on $\overline{\varphi(\Omega)}$. This means that $\left\|C_{\varphi}\left(f_{n}\right)\right\|_{\infty}=\left\|f_{n} \circ \varphi\right\|_{\infty} \rightarrow 0$, implying $\left\|f_{n} \circ \varphi\right\|_{2} \rightarrow 0$ and the compactness of $C_{\varphi}$.

Actually, $C_{\varphi}$ is compact on every Hardy space $H^{p}(\Omega), 1 \leq p \leq \infty$. This observation will be useful later on.

For 2), we may indeed dispense ourselves with the invariance of $a$, and force $a=0$ to be a fixed point of $\varphi$. Indeed, we can replace $\varphi$ by $\psi=\Psi_{b} \circ \varphi \circ \Psi_{a}$ where $b=\varphi(a)$ is arbitrary, and use Lemma 2.6 as well as the ideal property of compact linear operators. We set $A=\varphi^{\prime}(0)$. Expanding each coordinate $\varphi_{j}$ of $\varphi$ in a series of homogeneous polynomials, we may write $($ since $\varphi(0)=0)$ :

$$
\varphi(z)=\sum_{|\alpha|=1} c_{\alpha} z^{\alpha}+\sum_{s=2}^{\infty}\left(\sum_{|\alpha|=s} c_{\alpha} z^{\alpha}\right)=A(z)+\sum_{s=2}^{\infty}\left(\sum_{|\alpha|=s} c_{\alpha} z^{\alpha}\right),
$$

where $c_{\alpha}=\left(c_{\alpha, j}\right)_{j \geq 1} \in \mathbb{C}^{\infty}$. We clearly have (looking at the Fourier series of $\left.\varphi\left(z \mathrm{e}^{i \theta}\right)\right)$ :

$$
\|z\|_{1}<1 \quad \Longrightarrow \quad z \in \Omega \quad \Longrightarrow \quad A(z)=\frac{1}{2 \pi} \int_{0}^{2 \pi} \varphi\left(z \mathrm{e}^{i \theta}\right) \mathrm{e}^{-i \theta} d \theta .
$$

Since $\varphi$ is compact, this clearly implies, with $B$ the open unit ball of $\ell^{1}$, that $A(B)$ is totally bounded, proving the compactness of $A$.

The following extension of results of [11], then [1] and [6], which themselves extend a theorem of G. Königs ([14], p. 93) will play an essential role for lower bounds of approximation numbers. 
Theorem 3.3. Let $\varphi: \Omega \rightarrow \Omega$ be a compact symbol. Assume there is a $\in \Omega$ such that $\varphi(a)=a$ and that $\varphi^{\prime}(a) \in \mathcal{L}\left(\ell^{1}\right)$ is injective. Then, the spectrum of $C_{\varphi}: H^{2}(\Omega) \rightarrow H^{2}(\Omega)$ is exactly formed by the numbers $\lambda^{\alpha}, \alpha \in \mathbb{N}^{(\infty)}$, and 0,1 , where $\left(\lambda_{j}\right)_{j \geq 1}$ denote the eigenvalues of $A:=\varphi^{\prime}(a)$ and:

$$
\lambda^{\alpha}=\prod_{j \geq 1} \lambda_{j}^{\alpha_{j}} \quad \text { if } \quad \alpha=\left(\alpha_{j}\right)_{j \geq 1} \in \mathbb{N}^{(\infty)} .
$$

Proof. This is proved in [1 for the unit ball $B_{E}$ of an arbitrary Banach space $E$ and for the space $H^{\infty}\left(B_{E}\right)$, in four steps which are the following:

1. If $\varphi\left(B_{E}\right)$ lies strictly inside $B_{E}$ (namely if $\varphi\left(B_{E}\right) \subseteq r B_{E}$ for some $r<1$ ), in particular when $\varphi$ is compact, $\varphi$ has a unique fixed point $a \in B_{E}$, according to a theorem of Earle and Hamilton.

2. The spectrum of $C_{\varphi}$ contains the numbers $\lambda$ where $\lambda$ is an eigenvalue of $\varphi^{\prime}(a)$ or $\lambda=0,1$.

3. It is then proved that the spectrum of $C_{\varphi}$ contains the numbers $\lambda^{\alpha}$ and 0,1 .

4. It is finally proved that spectrum of $C_{\varphi}$ is contained in the numbers $\lambda^{\alpha}$ and 0,1 .

Here, handling with the domain $\Omega$, we see that:

1. True or not for $\Omega$, the Earle-Hamilton theorem is not needed since we will force, by a change of the compact symbol $\varphi$ in another compact symbol $\psi=\Psi_{b} \circ \varphi \circ \Psi_{a}$, the point 0 to be a fixed point. Moreover $A=\psi^{\prime}(0)$ is injective if $\varphi^{\prime}(a)$ is, since $\Psi_{a}^{\prime}$ and $\Psi_{b}^{\prime}$ are invertible.

2. The second step (non-surjectivity) is valid for any domain and for $H^{2}(\Omega)$, or $H^{p}(\Omega)$, in exactly the same way.

3. The third step consists of proving $\left\{\lambda^{\alpha}\right\} \subseteq \sigma\left(C_{\varphi}\right)$.

For that purpose, assume that $\lambda^{\alpha}=\prod_{l=1}^{m} \lambda_{l} \neq 0$ with $\lambda_{l}$ an eigenvalue of $\varphi^{\prime}(0)$ and with repetitions allowed. As we already mentioned, under the assumption of compactness of $\varphi, C_{\varphi}$ is compact on $H^{p}(\Omega)$ as well, for any $p \geq 1$. We take here $p=2 m$. Step 2 provides us with non-zero functions $f_{i} \in H^{p}(\Omega)$ such that $f_{i} \circ \varphi=\lambda_{i} f_{i}, 1 \leq i \leq m$, since for the compact operator $C_{\varphi}: H^{p} \rightarrow H^{p}$, non-surjectivity implies non-injectivity. Let $f=\prod_{1<i<m} f_{i}$. Then, using the integral representation of the norm and the Hölder inequality, we see that $f \in H^{2}(\Omega), f \neq 0$ and $f \circ \varphi=\lambda^{\alpha} f$, proving our claim.

4. The fourth step is valid as well, with a slight simplification: we have to show that, if $\mu \neq 1$ is not of the form $\lambda^{\alpha}$, then $C_{\varphi}-\mu I$ is injective. Let $f \in H^{2}(\Omega)$ satisfying $f \circ \varphi=\mu f$ and let:

$$
f(z)=\sum_{m=0}^{\infty} \frac{d^{m} f(0)}{m !}\left(z^{m}\right)
$$

be the Taylor expansion of $f$ about $z=0$ (observe that $\Omega$ is a Reinhardt domain). As usual, $d^{m} f(0)=: L_{m}$ is an $m$-linear symmetric form on $F=\ell^{1}$ and the notation $L_{m}\left(z^{m}\right)$ means $L_{m}(z, z, \ldots, z)$. 
Observe that $L_{m}$ can be isometrically identified with an element (denoted $\left.\overline{L_{m}}\right)$ of $\mathcal{L}\left(F^{\otimes n}\right)$ defined by the formula:

$$
\overline{L_{m}}\left(x_{1} \otimes \cdots \otimes x_{n}\right)=L_{m}\left(x_{1}, \ldots, x_{m}\right) .
$$

We will prove by induction that $L_{n}=0$ for each $n$. For this, we can avoid the appeal to transposes of [1] as follows: if the result holds for $L_{m}$ with $m<n$, one gets (comparing the terms in $z^{n}$ in both members of $f \circ \varphi=\mu f$ ):

$$
\mu A=A \circ B \quad \text { where } A=\overline{L_{n}} \text { and } B=\varphi^{\prime}(0)^{\otimes n} .
$$

That is $A(B-\mu I)=0$ where $I$ is the identity map of $F^{\otimes n}$. Now, $B-\mu I$ in invertible in $\mathcal{L}(F)$ by Lemma 3.3. so that $A=A(B-\mu I)(B-\mu I)^{-1}=0$.

The proof is complete.

The following theorem summarizes and exploits the preceding theorem. Possibly, some restrictions can be removed, and we could only assume the compactness of $C_{\varphi}$, not of $\varphi$ itself. After all, in dimension one, there are symbols $\varphi$ with $\|\varphi\|_{\infty}=1$ for which $C_{\varphi}: H^{2} \rightarrow H^{2}$ is compact.

Theorem 3.4. Let $\varphi: \Omega \rightarrow \Omega$ be a truly infinite-dimensional compact mapping of $\Omega$. Then:

1) $C_{\varphi}: H^{2}(\Omega) \rightarrow H^{2}(\Omega)$ is bounded and even compact.

2) $A=\varphi^{\prime}(0)$ is compact.

3) No $\delta>0$ can exist such that $a_{n}\left(C_{\varphi}\right) \leq C \mathrm{e}^{-c n^{\delta}}$ for all $n \geq 1$. More precisely, the numbers $a_{n}$ satisfy:

$$
\sum_{n \geq 1} \frac{1}{\log ^{p}\left(1 / a_{n}\right)}=\infty \text { for all } p<\infty
$$

Proof. The proof is based on the previous Theorem 3.3 Without loss of generality, we can assume that $\varphi(0)=0$ and $\varphi^{\prime}(0)$ is injective, by using a point $a$ at which $\varphi^{\prime}(a)$ is injective, and then the fact that automorphisms of $\Omega$ act transitively on $\Omega$, act boundedly on $H^{2}(\Omega)$, and the ideal property of approximation numbers. More precisely, we pass to $\Psi=\Psi_{b} \circ \varphi \circ \Psi_{a}$ with $b=\varphi(a)$ and get:

$$
\Psi(0)=0 \quad \text { and } \quad \Psi^{\prime}(b)=\Psi_{b}^{\prime}(b) \varphi^{\prime}(a) \Psi_{a}^{\prime}(0)
$$

injective, since $\Psi_{b}^{\prime}(b)$ and $\Psi_{a}^{\prime}(0)$ are, and $\Psi_{a}$ and $\Psi_{b}$ are automorphisms of $\Omega$.

We now have the following simple but crucial lemma.

Lemma 3.5. Whatever the choice of the numbers $\lambda_{j}$ with $0<\left|\lambda_{j}\right|<1$, denoting by $\left(\delta_{n}\right)_{n \geq 1}$ the non-increasing rearrangement of the numbers $\lambda^{\alpha}$, one has:

$$
\sum_{n \geq 1} \frac{1}{\log ^{p}\left(1 / \delta_{n}\right)}=\infty \text { for all } p<\infty
$$


Proof of the Lemma. For any positive integer $p$, we set:

$$
q=2 p, \quad \log 1 /\left|\lambda_{j}\right|=A_{j},
$$

and we use that:

$$
\sum_{1 \leq j \leq q} \alpha_{j} A_{j} \leq\left(\sum_{1 \leq j \leq q} \alpha_{j}^{2}\right)\left(\sum_{1 \leq j \leq q} A_{j}^{2}\right)=: C_{q}\left(\sum_{1 \leq j \leq q} \alpha_{j}^{2}\right)=C_{q}\|\alpha\|^{2},
$$

where $\|\cdot\|$ stands for the euclidean norm in $\mathbb{R}^{q}$. We then get:

$$
\begin{aligned}
\sum_{n \geq 1} \frac{1}{\log ^{p}\left(1 / \delta_{n}\right)} & =\sum_{\alpha>0} \frac{1}{\log ^{p}\left(1 /\left|\lambda^{\alpha}\right|\right)} \\
& \geq \sum_{\alpha_{j} \geq 1,1 \leq j \leq q} \frac{1}{\log ^{p}\left(1 /\left|\lambda_{1}^{\alpha_{1}}\right| \cdots 1 /\left|\lambda_{q}^{\alpha_{q}}\right|\right)} \\
& =\sum_{\alpha_{j} \geq 1,1 \leq j \leq q} \frac{1}{\left(\alpha_{1} A_{1}+\cdots+\alpha_{q} A_{q}\right)^{p}} \\
& \geq C_{q}^{-p} \sum_{\alpha_{j} \geq 1,1 \leq j \leq q} \frac{1}{\left(\alpha_{1}^{2}+\cdots+\alpha_{q}^{2}\right)^{p}} \\
& =C_{q}^{-p} \sum_{\alpha_{j} \geq 1,1 \leq j \leq q} \frac{1}{\|\alpha\|^{q}}=\infty,
\end{aligned}
$$

because:

$$
\int_{x \in \mathbb{R}^{q},\|x\| \geq 1} \frac{1}{\|x\|^{q}} d x=c_{q} \int_{1}^{\infty} \frac{r^{q-1}}{r^{q}} d r=\infty .
$$

This proves the lemma.

This can be transferred to the approximation numbers $a_{n}=a_{n}\left(C_{\varphi}\right)$ to end the proof of Theorem 3.4. Indeed, we know from Lemma 3.5 that the nonincreasing rearrangement $\left(\delta_{n}\right)$ of the eigenvalues $\lambda^{\alpha}$ of $C_{\varphi}$ satisfies

$$
\sum_{n \geq 1} \frac{1}{\log ^{p}\left(1 / \delta_{n}\right)}=\infty
$$

Since a divergent series of non-negative and non-increasing numbers $u_{n}$ satisfies $\sum u_{2 n}=\infty$, we further see that:

$$
\sum_{n \geq 1} \frac{1}{\log ^{p}\left(1 / \delta_{2 n}\right)}=\infty \text { for all } p<\infty
$$

Moreover, by Theorem 2.1 we have:

$$
\left(\frac{1}{2 \log 1 / \delta_{2 n}}\right)^{p} \leq\left(\frac{1}{\log 1 /\left(a_{1} a_{n}\right)}\right)^{p}
$$


Since $1 /\left(\log 1 / a_{1} a_{n}\right) \sim 1 /\left(\log 1 / a_{n}\right)$, Lemma 3.5 then gives the result. This clearly prevents an inequality of the form $a_{n} \leq C \mathrm{e}^{-c n^{\delta}}$ for some positive numbers $c, C, \delta$ and all $n \geq 1$. Indeed, this would imply:

$$
\sum_{n \geq 1} \frac{1}{\log ^{p}\left(1 / a_{n}\right)}<\infty \quad \text { for } \quad p>1 / \delta
$$

contradicting (3.3).

Remarks. Let us briefly comment on the assumptions in Theorem 3.4.

1) We do not need the Earle-Hamilton theorem under our assumptions. The Schauder-Tychonoff theorem gives the existence (if not the uniqueness) of a fixed point for $\varphi$ in $\Omega$ (bounded and convex).

2) The Earle-Hamilton theorem is in some sense more general (for analytic maps) since it remains valid when $\overline{\varphi(\Omega)}$ is only assumed to lie strictly inside $\Omega$, i.e. when $\varphi(\Omega) \subseteq r \Omega$ for some $r<1$. But this assumption does not ensure the compactness of $C_{\varphi}$ as indicated by the simple example $\varphi(z)=r z, 0<r<1$. The coordinate functions $z \mapsto z_{n}$ converge weakly to 0 , while $\left\|C_{\varphi}\left(z_{n}\right)\right\|_{H^{2}(\Omega)}=$ $r$.

3) The mere assumption that $\overline{\varphi(\Omega)}$ is compact is not sufficient either. Juste take:

$$
\varphi(z)=\left(\frac{1+z_{1}}{2}, 0, \ldots, 0, \ldots\right) .
$$

Since the composition operator $C_{\varphi_{1}}$ associated with $\varphi_{1}(z)=\frac{1+z}{2}$ is notoriously non-compact on $H^{2}(\mathbb{D})$, neither is $C_{\varphi}$ on $H^{2}(\Omega)$. Yet, $\overline{\varphi(\Omega)}$ is obviously compact in $\ell^{1}$.

\section{Possible upper bounds}

Recall that $\Omega=\mathbb{D}^{\infty} \cap \ell^{1}$.

\subsection{A general example}

Theorem 4.1. Let $\varphi\left(\left(z_{j}\right)_{j}\right)=\left(\lambda_{j} z_{j}\right)_{j}$ with $\left|\lambda_{j}\right|<1$ for all $j$, so that $\varphi(\Omega) \subseteq \Omega$ and $\varphi^{\prime}(0)$ is the diagonal operator with eigenvalues $\lambda_{j}, j \geq 1$, on the canonical basis of $\ell^{1}$. Let $p>0$. Then:

$$
\left(\lambda_{j}\right)_{j} \in \ell^{p} \quad \Longrightarrow \quad C_{\varphi} \in S_{p} .
$$

In particular, there exist truly infinite-dimensional symbols on $\Omega$ such that the composition operator $C_{\varphi}: H^{2}(\Omega) \rightarrow H^{2}(\Omega)$ is in all Schatten classes $S_{p}$, $p>0$. 
Proof. Since $C_{\varphi}$ is diagonal on the orthonormal basis $\left(z^{\alpha}\right)_{\alpha}$ of the Hilbert space $H^{2}(\Omega)$, with $C_{\varphi}\left(z^{\alpha}\right)=\varphi^{\alpha}$, its approximation numbers are the non-increasing rearrangement of the moduli of eigenvalues $\lambda^{\alpha}$, so that an Euler product-type computation gives:

$$
\sum_{n=1}^{\infty} a_{n}^{p}=\sum_{\alpha \in E}\left|\lambda^{\alpha}\right|^{p}=\sum_{\alpha_{j} \in \mathbb{N}} \prod_{j \geq 1}\left|\lambda_{j}\right|^{p \alpha_{j}}=\prod_{j=1}^{\infty}\left(1-\left|\lambda_{j}\right|^{p}\right)^{-1}<\infty .
$$

To obtain $C_{\varphi} \in \bigcap_{p>0} S_{p}$, just take $\lambda_{n}=\mathrm{e}^{-n}$. This ends the proof.

\subsection{A sharper upper bound}

By making a more quantitative study, we can prove the following result.

Theorem 4.2. For any $0<\delta<1$, there exists a compact composition operator on $H^{2}(\Omega)$, with a truly infinite-dimensional symbol, such that, for some positive constants $c, C, b$, we have:

$$
a_{n}\left(C_{\varphi}\right) \leq C \exp \left(-c \mathrm{e}^{b(\log n)^{\delta}}\right) .
$$

Proof. Take the same operator $C_{\varphi}$ as in Theorem 4.1, with $\lambda_{n}=\mathrm{e}^{-A_{n}}$ where the positive numbers $A_{n}$ have to be adjusted. Its approximation numbers $a_{N}$ are then the non-increasing rearrangement of the sequence of numbers $\left(\varepsilon_{n}\right)_{n}:=$ $\left(\lambda^{\alpha}\right)_{\alpha}$. This suggests using a generating function argument, namely considering $\sum \varepsilon_{n} x^{n}$, but the rearrangement perturbs the picture. Accordingly, we follow a sligthly different route. Fix an integer $N \geq 1$ and a real number $r>0$. Observe that, following the proof of Theorem 4.1 ,

$$
N a_{N}^{r} \leq \sum_{n=1}^{N} a_{n}^{r} \leq \sum_{n=1}^{\infty} a_{n}^{r}=\prod_{n=1}^{\infty}\left(1-e^{-r A_{n}}\right)^{-1} .
$$

First, consider the simple example $A_{n}=n$. We get:

$$
N a_{N}^{r} \leq \eta\left(\mathrm{e}^{-r}\right)
$$

where $\eta$ is the Dedekind eta function (see [5]) given by:

$$
\eta(x)=\prod_{n=1}^{\infty}\left(1-x^{n}\right)^{-1}=\sum_{n=0}^{\infty} p(n) x^{n}, \quad|x|<1,
$$

where $p(n)$ is the number of partitions of the integer $n$. It is well-known ([5], Ch. 7 , p. 169) that $\eta\left(\mathrm{e}^{-r}\right) \leq \mathrm{e}^{D / r}$ with $D=\pi^{2} / 6$, so that:

$$
a_{N} \leq \exp \left(\frac{D}{r^{2}}-\frac{\log N}{r}\right) .
$$

Optimizing with $r=2 D / \log N$, we get:

$$
a_{N} \leq \exp \left(-c \log ^{2} N\right),
$$


with $c=1 / 4 D$. This is more precise than Theorem 4.1 .

We now show that if $A_{n}$ increases faster, we can achieve the decay of Theorem 4.2. As before, we get in general:

$$
a_{N} \leq \inf _{x>1}\left(\exp \left[x\left(\log F\left(x^{-1}\right)-\log N\right)\right]\right),
$$

where

$$
F(r)=\prod_{n=1}^{\infty}\left(1-\mathrm{e}^{-r A_{n}}\right)^{-1}
$$

We have:

$$
\log F(r)=\sum_{n=1}^{\infty}\left(\sum_{m=1}^{\infty} \frac{\mathrm{e}^{-r m A_{n}}}{m}\right)=\sum_{m=1}^{\infty} \frac{1}{m}\left(\sum_{n=1}^{\infty} \mathrm{e}^{-r m A_{n}}\right) .
$$

Now, take $A_{n}=\mathrm{e}^{n^{\alpha}}$ where $\alpha>0$ is to be chosen. We have:

$$
\sum_{n=1}^{\infty} \mathrm{e}^{-r m \mathrm{e}^{n^{\alpha}}} \leq \int_{0}^{\infty} \mathrm{e}^{-r m \mathrm{e}^{\mathrm{t}^{\alpha}}} d t=: I_{m}(r)
$$

Standard estimates now give, for $r<1$ :

$$
\begin{aligned}
I_{m}(r) & =\int_{1}^{\infty} \mathrm{e}^{-r m x} \frac{1}{\alpha}(\log x)^{\frac{1}{\alpha}-1} \frac{d x}{x}=\int_{r m}^{\infty} \mathrm{e}^{-y} \frac{1}{\alpha}\left(\log \frac{y}{r m}\right)^{\frac{1}{\alpha}-1} \frac{d y}{y} \\
& \lesssim\left(\log \frac{1}{r}\right)^{\frac{1}{\alpha}-1} \int_{r m}^{\infty} \mathrm{e}^{-y} \frac{d y}{y} \lesssim \mathrm{e}^{-r m}\left(\log \frac{1}{r}\right)^{\frac{1}{\alpha}}
\end{aligned}
$$

so that:

$$
\log F(r) \lesssim(\log 1 / r)^{\frac{1}{\alpha}} \sum_{m=1}^{\infty} m^{-1} \mathrm{e}^{-r m} \lesssim(\log 1 / r)^{\frac{1}{\alpha}+1} .
$$

Going back to (4.1), we get, for some constant $C>0$, and for $x=1 / r>1$ :

$$
a_{N} \leq C \exp \left[C x\left((\log x)^{\frac{1}{\alpha}+1}-\log N\right)\right] .
$$

Adjusting $x=x_{N}>1$ so as to have $(\log x)^{\frac{1}{\alpha}+1}=\log N-1$, that is:

$$
x_{N}=\exp \left[(\log (N / \mathrm{e}))^{\frac{\alpha}{\alpha+1}}\right] \text {, }
$$

we get $a_{N} \leq C \mathrm{e}^{-c x_{N}}$, which is the claimed result with $\delta=\alpha /(\alpha+1)$.

This $\delta$ can be taken arbitrarily in $(0,1)$ by choosing $\alpha$ suitable, and we are done.

Remark. Of course, $\delta=1$ is forbidden, because this would give $a_{n} \leq C \mathrm{e}^{-c n^{b}}$, implying:

$$
\sum_{n=1}^{\infty} \frac{1}{\left(\log 1 / a_{n}\right)^{p}} \lesssim \sum_{n=1}^{\infty} n^{-b p}<\infty
$$

for large $p$, and contradicting Theorem 3.4 . 


\section{References}

[1] R. Aron, P. Galindo and M. Lindström, Compact homomorphisms between algebras of analytic functions, Studia Math. 123(3) (1997), 235-247.

[2] F. Bayart, D. Li, H. Queffélec and L. Rodríguez-Piazza, Approximation numbers of composition operators on the Hardy and Bergman spaces of the ball and of the polydisk, Math. Proc. of the Cambridge Philos. Soc., to appear.

[3] B. Carl and I. Stephani. Entropy, compactness and the approximation of operators, Cambridge Tracts in Mathematics 98, Cambridge University Press, Cambridge (1990).

[4] S. Chae, Holomorphy and Calculus in Normed Spaces, Monographs and Textbooks in Pure and Applied Mathematics 92, Marcel Dekker (1985).

[5] K. Chandrasekharan, Arithmetical Functions, Grundlehren Math. Wiss., Band 167, Berlin, Springer (1970).

[6] D. Clahane, Spectra of compact composition operators over bounded symmetric domains, Integral Equations Operator Theory 51 (2005), 41-56.

[7] B. Cole and T. Gamelin, Representing measures and Hardy spaces for the infinite polydisk algebra, Proc. Lond. Math. Society 53 (1986), 112-142.

[8] C. Cowen, B. MacCluer, Composition Operators on Spaces of Analytic Functions, Studies in Advanced Mathematics, C.R.C. Press (1994).

[9] S. E. Elliott and M. T. Jury, Composition operators on Hardy spaces of a half plane, Bull. London Math. Soc. 44, no. 3 (2012), 489-495.

[10] P. Lefèvre, Generalized Essential Norm of Weighted Composition Operators on some Uniform Algebras of Analytic Functions, Integral Equations Operator Theory 63 (2009), 557-569.

[11] B. MacCluer, Spectra of compact composition operators on $H^{p}\left(B_{N}\right)$, Analysis 4 (1984), 87-103.

[12] V. Matache, Composition operators on Hardy spaces on a half-plane, Proc. Amer. Math. Soc. 127(5) (1999), 1483-1491.

[13] W. Rudin, Functional Analysis, Second edition, Internat. Ser. Pure Appl. Math., McGraw-Hill (1991).

[14] J. H. Shapiro, Composition Operators and Classical Function Theory, Universitext, Tracts in Mathematics, Springer-Verlag, New York (1993).

[15] J. H. Shapiro and W. Smith, Hardy spaces that support no compact composition operators, J. Funct. Anal. 205 no. 1 (2003), 62-89. 


\section{Daniel Li}

Univ. Artois, Laboratoire de Mathématiques de Lens (LML) EA 2462, \& Fédération CNRS Nord-Pas-de-Calais FR 2956

Faculté Jean Perrin, Rue Jean Souvraz, S.P. 18

F-62 300 LENS, FRANCE

daniel.li@euler.univ-artois.fr

Hervé Queffélec

Univ. Lille Nord de France, USTL

Laboratoire Paul Painlevé U.M.R. CNRS 8524 \& Fédération CNRS Nord-Pasde-Calais FR 2956

F-59 655 VILLENEUVE D'ASCQ Cedex, FRANCE

Herve.Queffelec@univ-lille1.fr

Luis Rodríguez-Piazza

Universidad de Sevilla, Facultad de Matemáticas, Departamento de Análisis Matemático \& IMUS

Apartado de Correos 1160

41080 SEVILLA, SPAIN

piazza@us.es 